\title{
Shoulder-hand syndrome and symmetrical arthralgia in patients with tubo-ovarian carcinoma
}

\author{
A. J. TAGGART, J. M. I. IVESON, AND V. WRIGHT \\ From the Rheumatism Research Unit, Leeds, and the York District Hospital
}

SUMMARY Two cases of bilateral shoulder-hand syndrome and symmetrical arthralgia are described in patients with tubo-ovarian carcinoma. Swelling and contracture of the hands continued to develop despite oral corticosteroid therapy, and one patient underwent surgery in an attempt to prevent the development of further deformity.

The shoulder-hand syndrome has been associated with a variety of pathological conditions, including some cases of underlying malignancy. ${ }^{1}$ Tumours of the lung and brain are most frequently linked with the syndrome, ${ }^{2}$ but isolated case reports have implicated neoplasms at other sites. ${ }^{3-5}$ There are just 2 reports $^{67}$ in which ovarian tumours have been associated with this rheumatic syndrome. This paper describes 2 cases of disabling shoulder-hand syndrome and symmetrical arthralgia which occurred in patients with tubo-ovarian carcinoma.

\section{Case reports}

CAS E 1

This 58-year-old woman presented in April 1980 with left-sided lower abdominal pain, which was found at laparotomy to be due to a well differentiated adenocarcinoma of the left fallopian tube. The tumour was adherent to nearby omentum, but there were no obvious peritoneal secondaries, so hysterectomy together with bilateral salpingo-oophorectomy was carried out.

The patient remained well without further treatment for several months, but in April 1981 she began to complain of pain and swelling of the hands and knees with discomfort in the elbows and ankles. These pains were associated with morning stiffness, and the hand swelling made it difficult for her to make a fist. On examination there was oedema and thickening of the hands and forearms, with tenosynovitis of the right middle finger and a dusky

Accepted for publication 14 July 1983.

Correspondence to Dr A. J. Taggart, Department of Rheumatology, York District Hospital, Wigginton Road, York YO3 7HE. red discoloration of the dorsa of both hands. None of the other joints were swollen or tender, but the movements of both shoulders were markedly restricted and there were slight flexion deformities at the elbows. There was no clinical evidence of systemic sclerosis or vena caval obstruction.

Investigations revealed a haemoglobin of $15 \cdot 7$ $\mathrm{g} / \mathrm{dl}$, leucocytes $5 \cdot 8 \times 10^{9} / 1$ and erythrocyte sedimentation rate (ESR) $28 \mathrm{~mm} / \mathrm{h}$. Tests for rheumatoid (RAHA) and antinuclear factor (ANF) were negative and the levels of muscle enzymes (creatine phosphokinase, lactic dehydrogenase, and aspartate transaminase) were within normal limits. There was no evidence of myositis or myopathy on electromyography, but nerve conduction responses were of low amplitude, particularly in the lower limbs. A technetium- $99 \mathrm{~m}$ bone scan was normal, but a biopsy of enlarged supraclavicular lymph nodes showed replacement by secondary adenocarcinoma. Chest $x$-ray, barium swallow, and meal were all normal.

Progress. Diuretics and oral corticosteroids (prednisolone $15 \mathrm{mg} /$ day) had no effect on the swelling of the hands and forearms. The patient complained of some dyspnoea and upper sternal chest pain, so a course of radiotherapy was given to the supraclavicular and paratracheal lymph nodes. In March 1982 her abdominal pain recurred, and a hard mass was palpated in the left iliac fossa. She continued to deteriorate and died in May.

CASE 2

This 42-year-old patient presented in July 1981 with abdominal pain and ascites due to peritoneal spread from a poorly differentiated adenocarcinoma of the ovary. Three weeks after laparotomy and peritoneal biopsy, and one week after the start of chemotherapy 
with treosulfan ( $1 \mathrm{~g}$ daily), she began to develop swelling of both hands with stiffness of the shoulders, hips, and knees. Cytotoxic therapy was discontinued but the symptoms progressed. In November she was seen by a rheumatologist, who noted that both hands were shiny and oedematous, with fixed flexion deformities of the fingers, particularly at the proximal interphalangeal (PIP) joints. There was tethering of the overlying skin, with early palmar and plantar fasciitis (Figs. 1a, 1b). Movements were restricted in both shoulders and the hips were painful on rotation.

She had a haemoglobin of $12.5 \mathrm{~g} / \mathrm{dl}$, leucocytes $6 \cdot 0 \times 10^{9} / 1$, and ESR $20 \mathrm{~mm} / \mathrm{h}$. RAHA and ANF tests were negative, while liver function tests, creatinine kinase, and serum complement were also normal. $X$-rays of the chest, shoulders, hands, and feet were normal except for the soft tissue swelling and deformity of the hands.

Progress. Physiotherapy and oral prednisolone (15 $\mathrm{mg}$ daily) failed to produce any improvement in the hands or shoulders. Injection of the shoulder joints with hydrocortisone was equally fruitless. The right hand became increasingly clawed, and in July 1982
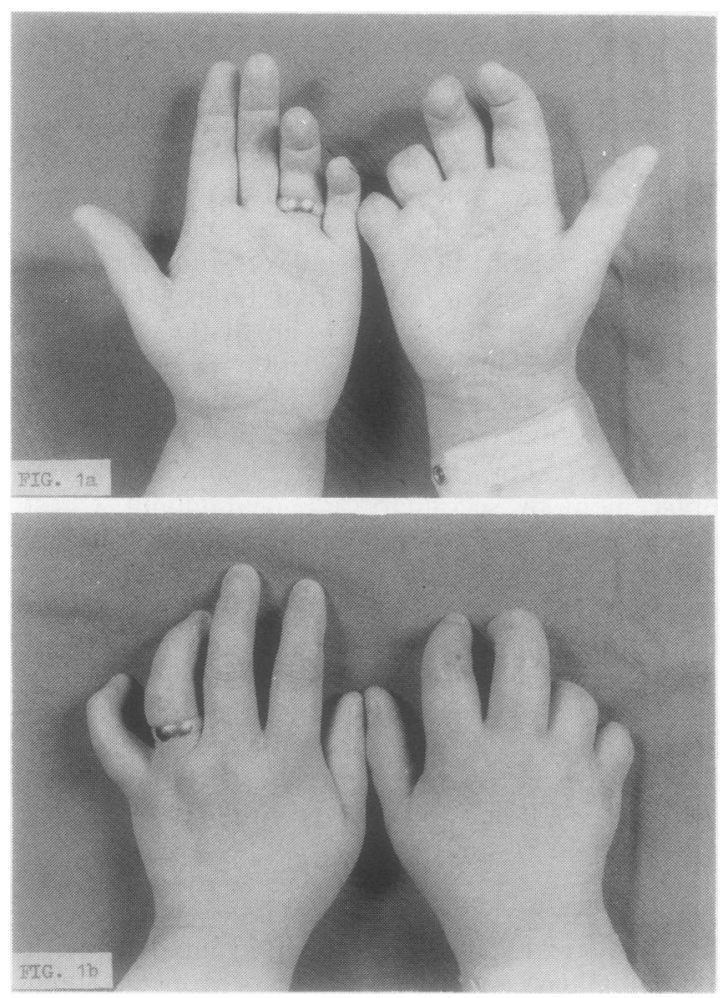

Figs. 1a, b. Hands of patient (case 2) after surgery to the right hand. Finger contractures, palmar fasciitis, and oedema of the hands are still evident. the palm was explored surgically. Soft tissue release failed to relieve the flexion contractures of the fingers, so the fourth and fifth digits were amputated at the PIP joints. The index and middle fingers were subsequently pinned at the PIP joints to prevent the development of further deformity. Biopsies of the palm and fingers demonstrated dense bands of regularly arranged fibrous tissue in the dermis with atrophy of the surrounding sweat glands. There was no evidence of vasculitis, myositis, or infiltration with neoplastic cells.

In October 1982 the patient was readmitted to hospital with a recurrence of her abdominal pain. $X$-rays of the hands showed disuse osteoporosis but no signs of erosive joint disease or periostitis. A technetium- $99 \mathrm{~m}$ bone scan confirmed the absence of bony metastases. Recurrence of the peritoneal secondaries was confirmed at laparotomy, and her progress followed a gradual downhill course. The patient died in March 1983 after several episodes of subacute intestinal obstruction.

\section{Discussion}

The striking clinical feature in both of these cases was the sclerodermatous appearance of the hands, but neither patient had signs of systemic sclerosis. The shoulder-hand syndrome is usually a self-limiting condition, but approximately $10 \%$ of patients go on to develop fibrosis and contracture of the hands which may easily be confused with scleroderma. ${ }^{689}$ Localised scleroderma is a recognised feature of some carcinoid tumours, ${ }^{10}$ but it is not associated with restriction of the shoulder movements. Both of these patients had evidence of bilateral frozen shoulders, and in case 2 palmar and plantar fasciitis was also a prominent feature.

Medsger et al. ${ }^{7}$ have recently described a similar association between palmar/plantar fasciitis, the shoulder-hand syndrome, and malignant tumours of the ovary. Some of their patients also had an inflammatory arthropathy of the large joints such as the knees. Both of our patients complained of pain and stiffness in the knees. In the first case the elbows and ankles were also affected, and in the second the hips were stiff and sore.

The shoulder-hand syndrome is associated with so many different conditions that it is sometimes difficult to ascribe it to a particular factor. Neither of these patients had any underlying condition normally associated with the syndrome, nor were they receiving drugs that are known to cause such a reaction. Although this is only the third report of an association between ovarian malignancy and the syndrome, it may not be as rare as expected. A retrospective survey of 169 cases of ovarian malignancy treated in 
this hospital in the past 5 years (1978-82) has uncovered a further case of the shoulder-hand syndrome in a patient with disseminated papillary adenocarcinoma of the ovary. Unfortunately the records in this case are incomplete and there is no indication whether arthritis or fasciitis were also features of the illness.

The aetiology and pathogenesis of the shoulderhand syndrome is not clear, but many workers have suggested that neurovascular reflexes play a role in its development. This may not be the only factor. Medsger et al. ${ }^{7}$ noted a marked degree of stromal fibrosis in the tumours of their patients and postulated that the neoplastic ovarian cells might be releasing a fibrosing substance into the systemic circulation. Oestrogens may be relevant here as in alcoholic liver disease, where they are thought to play a role in the pathogenesis of Dupuytren's contractures. Excessive fibrous tissue was present in the ovarian tumour from our second patient. Neoplasms of the fallopian tube may also have a fibrosing potential. It is interesting to note that there has been a report of retroperitoneal fibrosis occurring in a patient with this rare tumour. ${ }^{11}$

Malignancies of the ovary and fallopian tube are notoriously difficult to diagnose at an early stage, and these cases are a reminder that the shoulder-hand syndrome may be a systemic feature of these tumours. In both patients the diagnosis of malignancy had already been made, but rheumatic symptoms of this kind may sometimes precede the development of the abdominal symptoms by several months. ${ }^{67}$ The development of shoulder-hand syndrome in a woman who also complains of arthralgia should prompt a careful search for these tumours.

\section{References}

1 Steinbrocker O, Argyros T D. Shoulder-hand syndrome: present status as diagnostic and therapeutic entity. Med Clin North Am 1958; 42: 1533-53.

2 Steinbrocker $O$. The shoulder-hand syndrome-present perspective. Arch Phys Med Rehabil 1968; 49: 388-95.

3 DePalma A F. Loss of scapulohumeral motion (frozen shoulder). Ann Surg 1952; 135: 193-204.

4 Sheldon P J H. A retrospective survey of 102 cases of shoulder pain. Rheumatol Phys Med 1972; 11: 422-7.

5 Tyber M A. Treatment of the painful shoulder syndrome with amitriptyline and lithium carbonate. Can Med Assoc J 1974; 111: $137-40$.

6 Bremer C. Shoulder-hand syndrome; a case of unusual aetiology. Ann Phys Med 1966; 9: 168-71.

7 Medsger T, Dixon J A, Garwood V F. Palmar fasciitis and polyarthritis associated with ovarian carcinoma. Ann Intern Med 1982; 96: 424-31.

8 Wright V. The shoulder-hand syndrome. Rep Rheum Dis 1968; 24: $1-2$.

9 Woolf D. Shoulder-hand syndrome. Practitioner 1974; 213: 176-83.

10 Caldwell D S. Musculoskeletal syndromes associated with malignancy. Semin Arthritis Rheum 1981; 10: 198-223.

11 Anderson K A, Tanagho E A. Fallopian tube carcinoma associated with retroperitone al fibrosis. J Urol 1981; 125: 119-21. 